\title{
IncRNA SNHG1 attenuates osteogenic differentiation via the miR-101/DKK1 axis in bone marrow mesenchymal stem cells
}

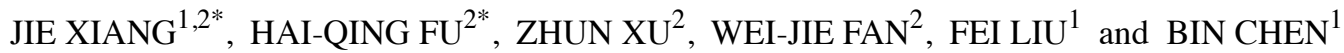 \\ ${ }^{1}$ Department of Orthopedics and Traumatology, Nanfang Hospital, Southern Medical University, \\ Guangzhou, Guangdong 510515; ${ }^{2}$ Department of Orthopedics and Traumatology, \\ The First Affiliated Hospital of University of South China, Hengyang, Hunan 421001, P.R. China
}

Received December 3, 2019; Accepted July 13, 2020

DOI: $10.3892 / \mathrm{mmr} .2020 .11489$

\begin{abstract}
The imbalance induced by inhibition of bone mesenchymal stem cell (BMSC) osteogenic differentiation results in osteoporosis $(\mathrm{OP})$; however, the underlying regulatory mechanism is not completely understood. Long non-coding RNAs (lncRNAs) serve crucial roles in osteogenic differentiation; therefore, investigating their regulatory role in the process of osteogenic differentiation may identify a promising therapeutic target for OP. The expression of small nucleolar RNA host gene 1 (SNHG1), Dickkopf 1 (DKK1), microRNA (miR)-101, RUNX family transcription factor 2 (RUNX2), osteopontin (OPN) and osteocalin (OCN) were detected via reverse transcription-quantitative PCR. The protein expression levels of DKK1, $\beta$-catenin, RUNX2, OPN, OCN, osterix and collagen type I $\alpha 1$ chain were analyzed by performing western blotting. The osteoblastic phenotype was assessed by conducting alkaline phosphatase activity detection and Alizarin Red staining. The interaction between SNHG1 and miR-101 was validated by bioinformatics and luciferase assays. The regulatory role of SNHG1 in BMSC osteogenic differentiation was assessed. SNHG1 expression was downregulated in a time-dependent manner during the process of osteogenic differentiation. SNHG1 overexpression inhibited osteogenic differentiation compared with the pcDNA group. The results indicated that SNHG1 and DKK1 directly interacted with miR-101. Moreover, SNHG1 regulated the $\mathrm{Wnt} / \beta$-catenin signaling pathway to inhibit osteogenic differentiation via the miR-101/DKK1 axis. The present study indicated that lncRNA
\end{abstract}

Correspondence to: Dr Bin Chen, Department of Orthopedics and Traumatology, Nanfang Hospital, Southern Medical University, 1838 Guangzhou Avenue North, Guangzhou, Guangdong 510515, P.R. China

E-mail: chenbinsurgeon@163.com

*Contributed equally

Key words: small nucleolar RNA host gene 1, microRNA-101, dickkopf WNT signaling pathway inhibitor 1, osteogenic differentiation
SNHG1 could attenuate BMSC osteogenic differentiation via the miR-101/DKK1 axis as a competitive endogenous RNA. Therefore, the present study furthered the current understanding of the potential mechanism underlying lncRNAs in in osteogenic differentiation.

\section{Introduction}

As a serious chronic systemic bone disease, osteoporosis (OP) involves excessive bone resorption and decreased bone formation (1), resulting from an imbalance induced by inhibition of osteogenic differentiation (2), such as abnormal bone mesenchymal stem cell (BMSC) differentiation, the precursor cells of osteoblasts (2). Due to their osteogenic differentiation ability, BMSCs were indicated to be a suitable cell type for the repair and remodeling of bones (3). Therefore, it is of significance to induce BMSC differentiation into bone tissues for OP treatment, and the mechanism to enhance osteogenic differentiation has received increasing attention.

Long non-coding RNAs (IncRNAs) are non-coding RNAs, $>200$ nucleotides in length, that regulate different processes via various molecular mechanisms (4). It has been reported that lncRNAs participate in regulating osteogenic activity. For instance, KCNQ1 opposite strand/antisense transcript 1 (KCNQ1OT1) regulates osteogenic differentiation by sponging microRNA (miRNA/miR)-214 (5), whereas MALAT1 promotes osterix (OSX) expression to regulate osteogenic differentiation by sponging miRNA-143 (6). Small nucleolar RNA host gene 1 (SNHG1) is upregulated in numerous types of cancer $(7,8)$, and can attenuate heterogeneous nuclear ribonucleoprotein $\mathrm{C}-\mathrm{p} 53$ protein interactions to enhance the stability of p53 (4). Besides, elevated SNHG1 can contribute to the progression of non-small cell lung cancer via inhibition of miR-101-3p (9). Moreover, SNHG1 inhibits BMSC osteogenic differentiation by modulating the $\mathrm{p} 38$ MAPK signaling pathway (10). However, the molecular mechanism underlying lncRNA SNHG1-mediated regulation of BMSC differentiation is not completely understood.

miRNAs are 18-25 base-long endogenous RNAs that are involved in regulating multiple physiological biological processes (11). miR-101 is significantly upregulated during human BMSC osteogenic differentiation, and it can target the enhancer of zeste 2 polycomb repressive complex 2 
subunit/Wnt/ $\beta$-catenin signaling pathway to promote human BMSC osteogenic differentiation (12). Bioinformatics analysis has revealed that SNHG1 has a miR-101 binding region (13); however, whether SNHG1 regulates BMSC osteogenic differentiation by binding with miR-101 is not completely understood.

The entire osteoblastic lineage is affected by Wnt signaling, and Wnt/ $\beta$-catenin signaling can indirectly repress osteoclast differentiation and bone resorption $(14,15)$. Dickkopf 1 (DKK1) is a Wnt signaling inhibitor, which inhibits the canonical Wnt signaling pathway by binding to the Wnt complex receptor Low-density lipoprotein receptor-related protein 5/6 (16). By bioinformatics, it was also predicted that there may be a binding site between miR-101 and DKK1 (17). Moreover, to the best of our knowledge, no previous study has focused on the regulation of BMSC osteogenic differentiation via the miR-101/DKK1/Wnt axis. It was hypothesized that the SNHG1/miR-101/DKK1 axis served a regulatory role in BMSC osteogenic differentiation.

\section{Materials and methods}

Cell culture. Human BMSCs (cat. no. HUXMA-01001; Cyagen Biosciences) were cultured in DMEM (HyClone; Cytiva) containing 10\% FBS (Gibco; Thermo Fisher Scientific, Inc.), $100 \mathrm{U} / \mathrm{ml}$ penicillin and $100 \mathrm{U} / \mathrm{ml}$ streptomycin. Cells were cultured in a humidified atmosphere containing $5 \% \mathrm{CO}_{2}$ at $37^{\circ} \mathrm{C}$.

Induction of osteogenic differentiation. To induce osteogenic differentiation, cells were cultured in osteogenic differentiation medium consisting of DMEM with $10 \%$ FBS, dexamethasone (100 nM; Sigma-Aldrich; Merck KGaA), ascorbic acid 2-phosphate (200 $\mu \mathrm{M}$; Sigma-Aldrich; Merck KGaA) and $\beta$-glycerophosphate (10 mM; Sigma-Aldrich; Merck KGaA) for 15 days at $37^{\circ} \mathrm{C}$. The medium was changed every 3 days.

Cell transfection. pcDNA-SNHG1and miR-101 mimics were constructed and purchased from Shanghai GenePharma Co., Ltd.. Cells were transfected with 200 ng/well pcDNA-SNHG1, pcDNA empty vector and $50 \mathrm{nM}$ miR-101 mimics (UAC AGUACUGUGAUAACUGAA) and its scramble control (mimics-NC) (UCACAACCUCCUAGAAAGAGUAGA) using Lipofectamine ${ }^{\circledR} 3000$ (Invitrogen; Thermo Fisher Scientific, Inc.) according to the manufacturer's protocol when the confluence of the cells reached $70-80 \%$. Then, cells were collected at $48 \mathrm{~h}$ after transfection for further use.

Luciferase activity assay. The predicted miR-101 binding site in SNHG1 and DKK1, as well as the mutated regions, were amplified and cloned into the pmirGLO plasmid (Promega Corporation). Then, $1 \times 10^{4} /$ well $293 \mathrm{~T}$ cells were co-transfected with $800 \mathrm{ng}$ luciferase reporter plasmid and $50 \mathrm{pmol}$ miRNA mimics or mimics-negative control (NC) using Lipofectamine 3000 in a 24 -well plate. At $48 \mathrm{~h}$ post-transfection, the Dual-Luciferase Reporter Assay system (Promega Corporation) was used to examine relative luciferase activity. Fluorescence signals were normalized to those of Renilla luciferase activity, and are presented as the mean \pm SD of three independent experiments.
Alkaline phosphatase (ALP) activity detection. ALP staining was performed using an ALP activity colorimetric assay kit (BioVision, Inc.) following the manufacturer's instructions. Briefly, cells were fixed with $70 \%$ ethanol at room temperature for $30 \mathrm{~min}$ and washed with PBST. Then, $50 \mu 15 \mathrm{mM}$ P-nitrophenyl phosphate (p-NPP) solution was added to test and control samples, and $10 \mu \mathrm{l}$ ALP enzyme solution was added to each p-NPP Standard wells and incubated at room temperature for 15-30 min. Cells were gently washed three times with cold PBS. Subsequently, cells were lysed with $1 \%$ Triton X-100 (Sigma-Aldrich; Merck KGaA) and washed with deionized water. The absorbance of p-nitrophenol at a wavelength of $405 \mathrm{~nm}$ was measured using a microplate reader (Bio-Rad Laboratories, Inc.) according to the manufacturer's instructions.

Alizarin red staining (ARS). ARS (Cyagen Biosciences, Inc.) was conducted to assess mineral deposition. Briefly, cells were fixed with $4 \%$ paraformaldehyde at room temperature for 15 min and stained with $2 \%$ Alizarin $\operatorname{Red~S~(pH~4.2;~}$ Sigma-Aldrich; Merck KGaA) at room temperature in deionized water for $20 \mathrm{~min}$. Subsequently, cells were washed with PBS and observed under a light microscope (Leica Microsystems $\mathrm{GmbH}$, magnification, x100).

Reverse transcription-quantitative PCR (RT-qPCR). Total RNA was extracted from cells using TRIzol ${ }^{\circledR}$ (Invitrogen; Thermo Fisher Scientific, Inc.). Total RNA was reverse transcribed into cDNA using the ImProm-II Reverse Transcription system (Promega Corporation) following the manufacturer's instructions. Briefly, the reaction was incubated at $37^{\circ} \mathrm{C}$ for $1 \mathrm{~h}$ followed by $95^{\circ} \mathrm{C}$ for $5 \mathrm{~min}$ and $4^{\circ} \mathrm{C}$ for $5 \mathrm{~min}$ Subsequently, qPCR was performed on days 0,7 and 15 of osteogenic differentiation using TransStart Eco Green qPCR SuperMix (TransGen Biotech Co., Ltd.) and the following thermocycling conditions: Initial denaturation at $95^{\circ} \mathrm{C}$ for $10 \mathrm{~min}$; 40 cycles of denaturation/annealing/extension at $95^{\circ} \mathrm{C}$ for $15 \mathrm{sec} ; 60^{\circ} \mathrm{C}$ for $30 \mathrm{sec}$ and followed by final extension at $60^{\circ} \mathrm{C}$ for $35 \mathrm{sec}$. The following primer sequences were used for qPCR: SNHG1 forward, 5'-ACTCCACTTCGTGTCTGT TCC-3' and reverse, 5'-TGAAGAGCAAGGCCCTGAAT-3'; miR-101 forward, 5'-GCGCGCATACAGTACTGTGATA-3' and reverse, 5'-GTCGTATCCAGTGCAGGGTCCGAGGTA TTCGCACTGGATACGACTTCAGT-3'; DKK1 forward, 5'-TGCCCCGGGAATTACTGCAAA-3' and reverse, 5'-CTG GAATACCCATCCAAGGTGCTA-3'; RUNX family transcription factor 2 (RUNX2) forward, 5'-CGGAATGCCTCT GCTGTTAT-3' and reverse, 5'-TTCCCGAGGTCCATCTAC TG-3'; osteopontin (OPN) forward, 5'-GATGGCCGAGGT GATAGTGT-3' and reverse, 5'-GTGGGTTTCAGCACTCTG GT-3'; osteocalin (OCN) forward, 5'-GGCAGCGAGGTAGTG AAGAG-3' and reverse, 5'-CTAGACCGGGCCGTAGAAG-3'; GAPDH forward, 5'-CCAGGTGGTCTCCTCTGA-3' and reverse, 5'-GCTGTAGCCAAATCGTTGT-3'; and U6 forward, 5'-CTCGCTTCGGCAGCACA-3' and reverse, 5'-AACGCT TCACGAATTTGCGT-3'. miRNA and mRNA expression levels were quantified using $2^{-\Delta \Delta C q}$ method and normalized to the internal reference genes U6 and GAPDH, respectively (18).

Western blotting. Total protein was extracted from cells using RIPA lysis buffer containing protease (Beyotime Institute of 

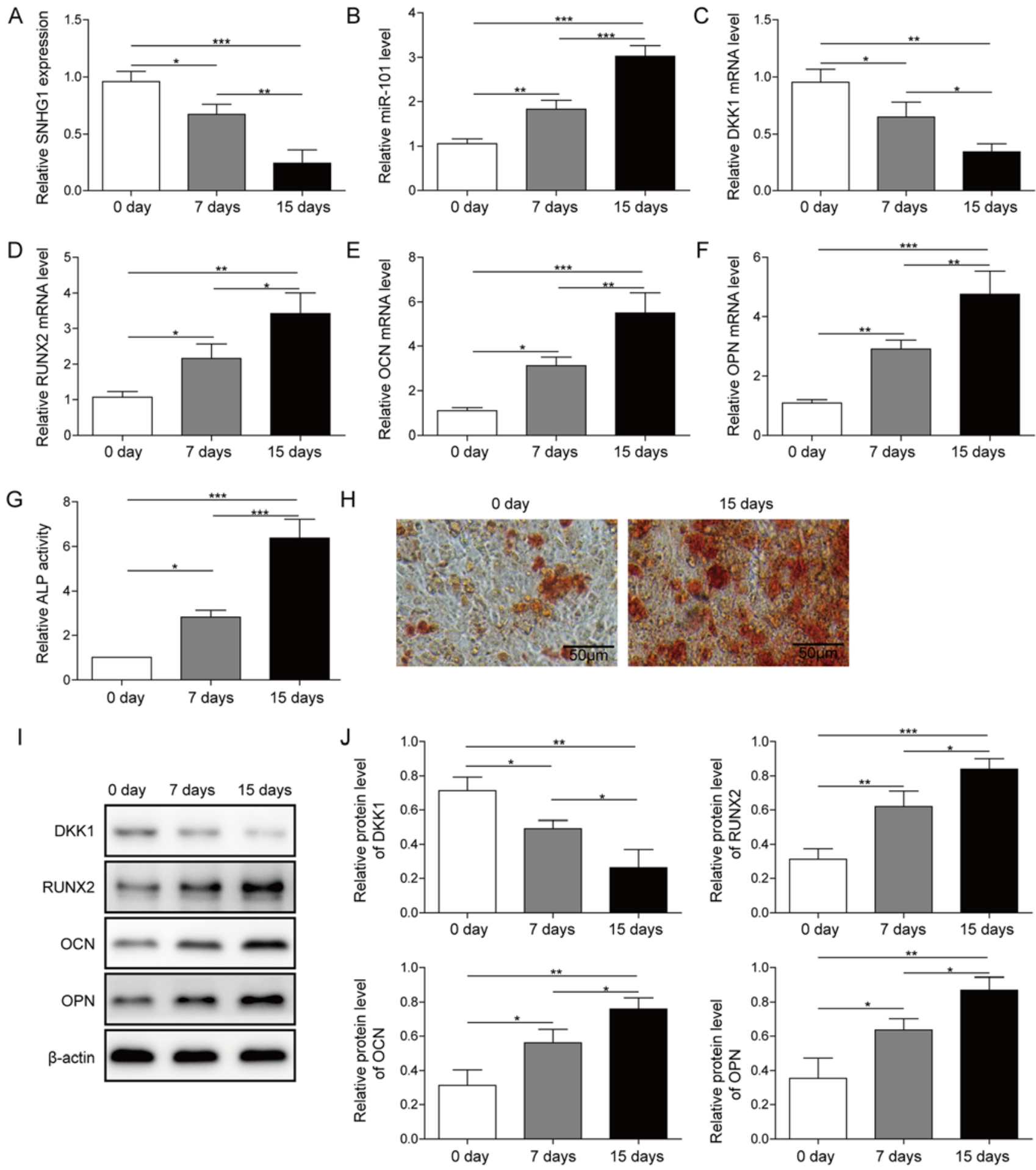

Figure 1. Expression of SNHG1, DKK1 and miR-101 during osteogenic differentiation. Expression of (A) SNHG1, (B) miR-101, (C) DKK1, (D) RUNX2, (E) OCN and (F) OPN in osteogenic differentiation medium-treated BMSCs at different time points. (G) ALP activity and (H) Alizarin Red staining in osteogenic differentiation medium-treated BMSCs at different time points. Protein expression levels were (I) determined by western blotting and (J) semi-quantified for DKK1, RUNX2, OPN and OCN in osteogenic differentiation medium-treated BMSCs at different time points. ${ }^{*} \mathrm{P}<0.05,{ }^{* *} \mathrm{P}<0.01$ and ${ }^{* * *} \mathrm{P}<0.001$. SNHG1, small nucleolar RNA host gene 1; DKK1, dickkopf WNT signaling pathway inhibitor 1; miR, microRNA; RUNX2, RUNX family transcription factor 2; OCN, osteocalin; OPN, osteopontin; ALP, alkaline phosphatase; BMSC, bone mesenchymal stem cell; d, days.

Biotechnology). Total protein was quantified using a BCA protein assay (Thermo Fisher Scientific, Inc.). A total of $100 \mu \mathrm{g}$ protein/lane was separated via 10\% SDS-PAGE and transferred to nitrocellulose membranes. After blocking with TBST buffer containing 5\% non-fat milk (Thermo Fisher Scientific, Inc.) for $1 \mathrm{~h}$ at room temperature, the membranes were incubated with specific primary antibodies at $4{ }^{\circ} \mathrm{C}$ overnight targeted against: DKK1 (1:1,000; cat. no. ab109416, Abcam), $\beta$-catenin (1:1,000; cat. no. ab184919; Abcam), RUNX2 (1:1,000; cat. no. ab192256; Abcam), OCN (1:1,000; cat. no. ab133612; Abcam), OPN (1:1,000; cat. no. ab214050; Abcam), OSX (1:500; cat. no. ab209484; Abcam), collagen type I $\alpha 1$ chain 

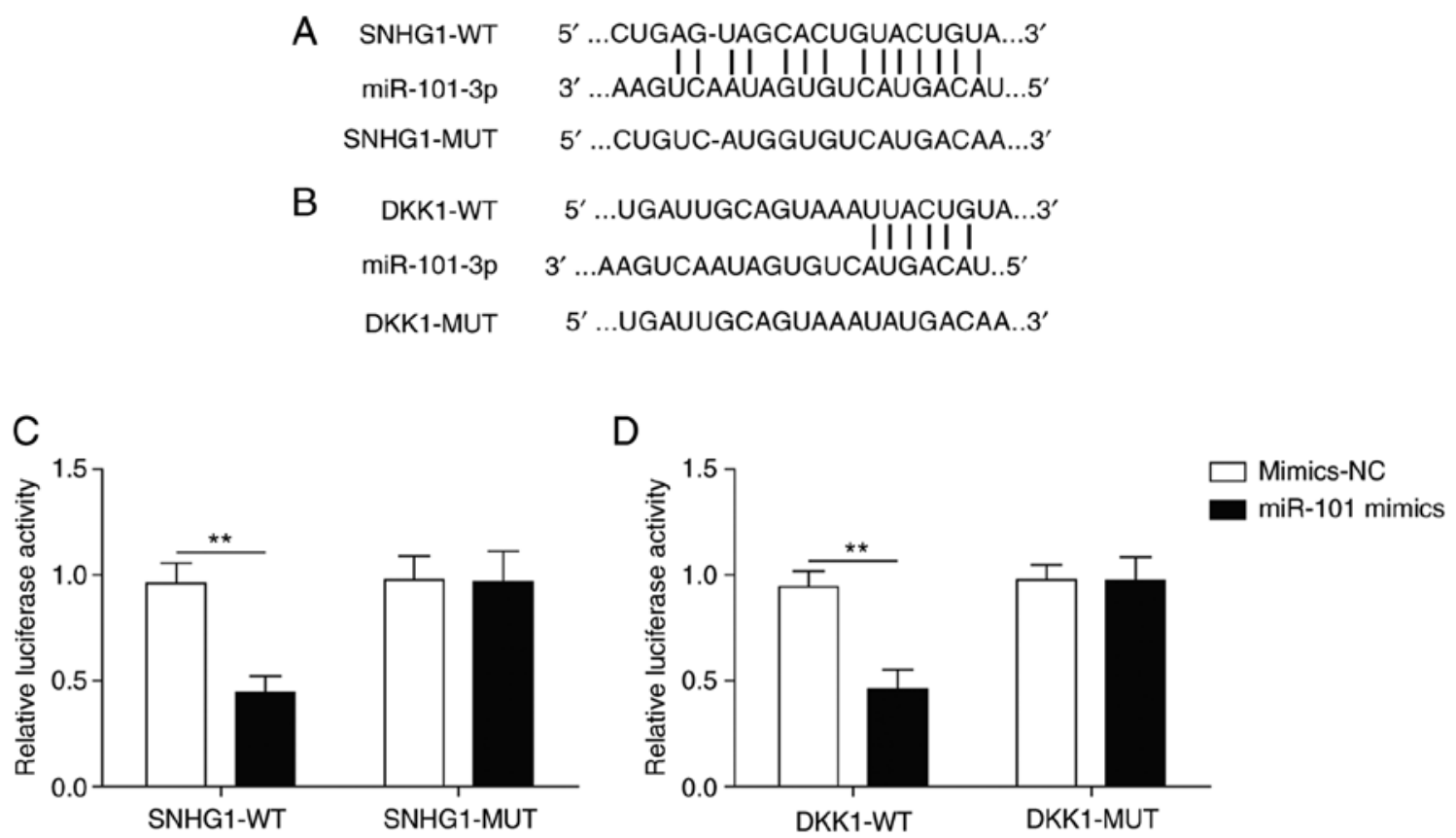

Figure 2. SNHG1 and DKK1 directly bind to miR-101. Potential miR-101 binding sites in (A) SNHG1 and (B) DKK1 mRNA were predicted by bioinformatics analysis. The effect of miR-101 mimics on the luciferase activity of (C) SNHG1-WT, SNHG1-MUT, (D) DKK1-WT and DKK1-MUT. .** P 0.01 . SNHG1, small nucleolar RNA host gene 1; DKK1, dickkopf WNT signaling pathway inhibitor 1; miR, microRNA; WT, wild-type; MUT, mutated; NC, negative control.

(COL1a1; 1:1,000; cat. no. ab34710; Abcam) and $\beta$-actin (1:1,000; cat. no. ab8227; Abcam). Following primary incubation, the membranes were incubated with goat anti-Rabbit IgG horseradish-peroxidase-conjugated secondary antibodies (1:5,000; cat. no. G-21234; Thermo Fisher Scientific, Inc.) for $1 \mathrm{~h}$ at room temperature. Protein bands were visualized using chemiluminescence ECL detection system (EMD Millipore) and the Bio-Rad XRS chemiluminescence detection system (Bio-Rad Laboratories, Inc.). $\beta$-actin was used as the loading control. The proteins were quantified using Quantity One software v4.62 (Bio-Rad Laboratories, Inc.).

Statistical analysis. Data are presented as the mean \pm standard deviation. The unpaired Student's t-test was used to compare the difference between two groups. One-way ANOVA followed by Tukey's post hoc test was used to compare differences among multiple groups. Statistical analyses were conducted using SPSS software (version 18.0; SPSS, Inc.). P $<0.05$ was considered to indicate a statistically significant difference. All experiments were performed in triplicate and carried out at least three times.

\section{Results}

Expression levels of SNHG1, miR-101 and DKK1 during BMSC osteogenic differentiation. After induction of osteogenic differentiation, the expression of SNHG1, miR-101, DKK1 and osteogenesis-related genes, including RUNX2, OCN and OPN, on day 0, 7 and 15 were detected via RT-qPCR. During the process of osteogenic differentiation, the expression of miR-101 and the osteogenesis-related genes were upregulated, whereas SNHG1 and DKK1 were downregulated in a time-dependent manner (Fig. 1A-F). Moreover, ALP activity and mineralization assays indicated an osteoblastic phenotype (Fig. 1G and H). Furthermore, the protein expression levels of the osteogenesis-related genes were also upregulated in a time-dependent manner during the process of osteogenic differentiation, whereas DKK1 protein expression levels were downregulated in a time-dependent manner (Fig. 1I and J). Collectively, the results indicated that SNHG1, miR-101 and DKK1 served a regulatory role in osteogenic differentiation.

SNHG1 and DKK1 are targets of miR-101. Firstly, the binding sites of miR-101 on SNHG1 (Fig. 2A) and DKK1 (Fig. 2B) were predicted by bioinformatics analysis. The luciferase assay indicated that miR-101 mimics significantly decreased the luciferase activity of SNHG1-wild-type (WT) compared with mimics-NC, but miR-101 mimics did not significantly alter the luciferase activity of SNHG1-mutated (MUT), indicating a direct interaction between SNHG1 and miR-101 (Fig. 2C). Similarly, miR-101 mimics significantly decreased the luciferase activity of DKK1-WT compared with mimics-NC, but did not significantly alter the luciferase activity of DKK1-MUT (Fig. 2D). The results demonstrated that SNHG1 and DKK1 were targets of miR-101.

SNHG1 inhibits BMSC osteogenic differentiation. Compared with pcDNA, SNHG1 overexpression significantly increased the expression of SNHG1 and DKK1 (Fig. 3A), but significantly decreased the expression of miR-101 (Fig. 3A). The expression levels of the osteogenesis-related markers were also significantly decreased by SNHG1 overexpression compared with pcDNA (Fig. 3B). Meanwhile, ALP activity and matrix mineralization were both obviously decreased by SNHG1 overexpression compared with pcDNA (Fig. 3C and D). Compared with pcDNA, the protein expression level of DKK1 was significantly increased, whereas the protein expression levels of the osteogenesis-related markers were significantly decreased after SNHG1 overexpression (Fig. 3E and F), which 

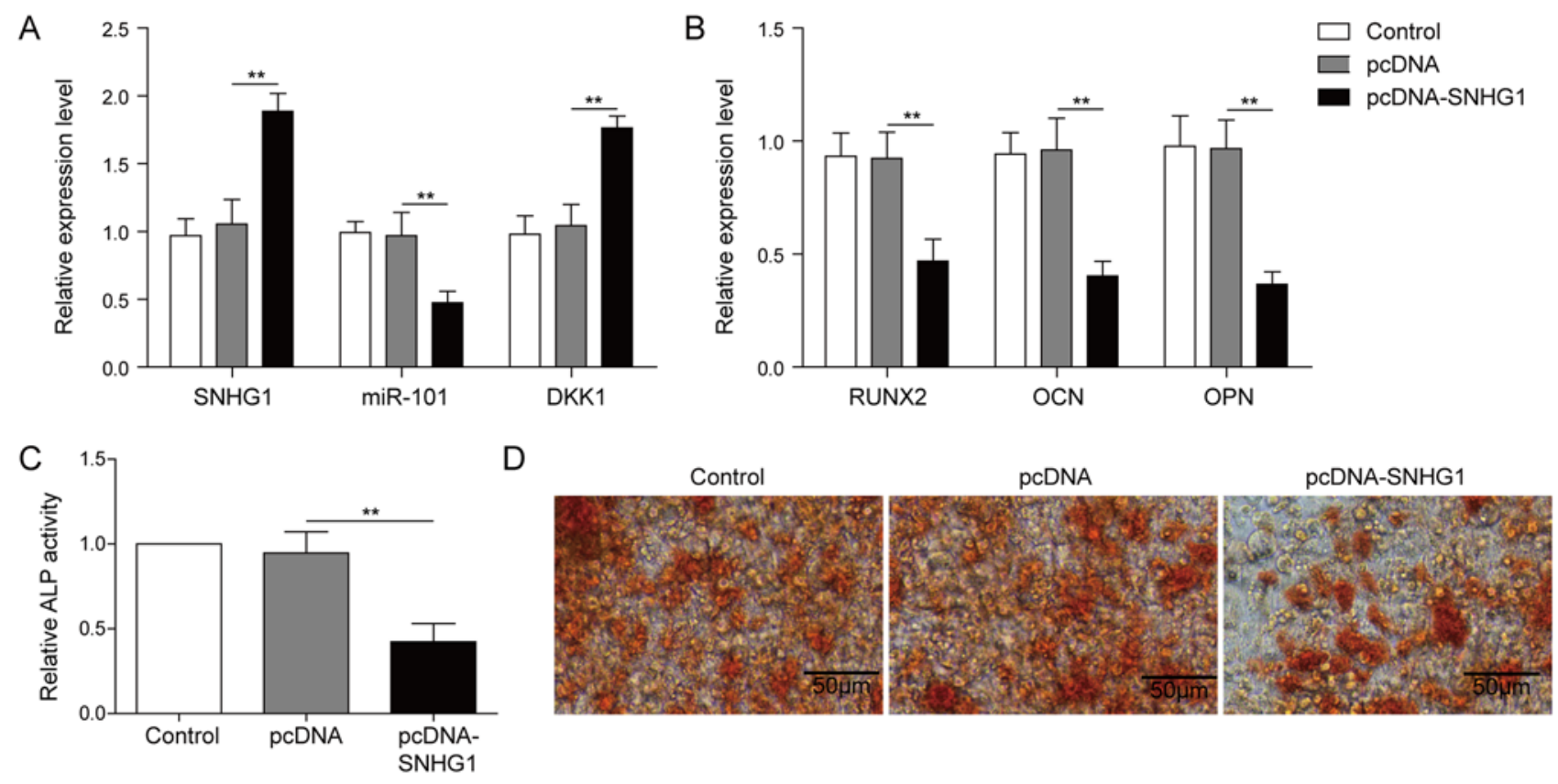

$\mathrm{E}$
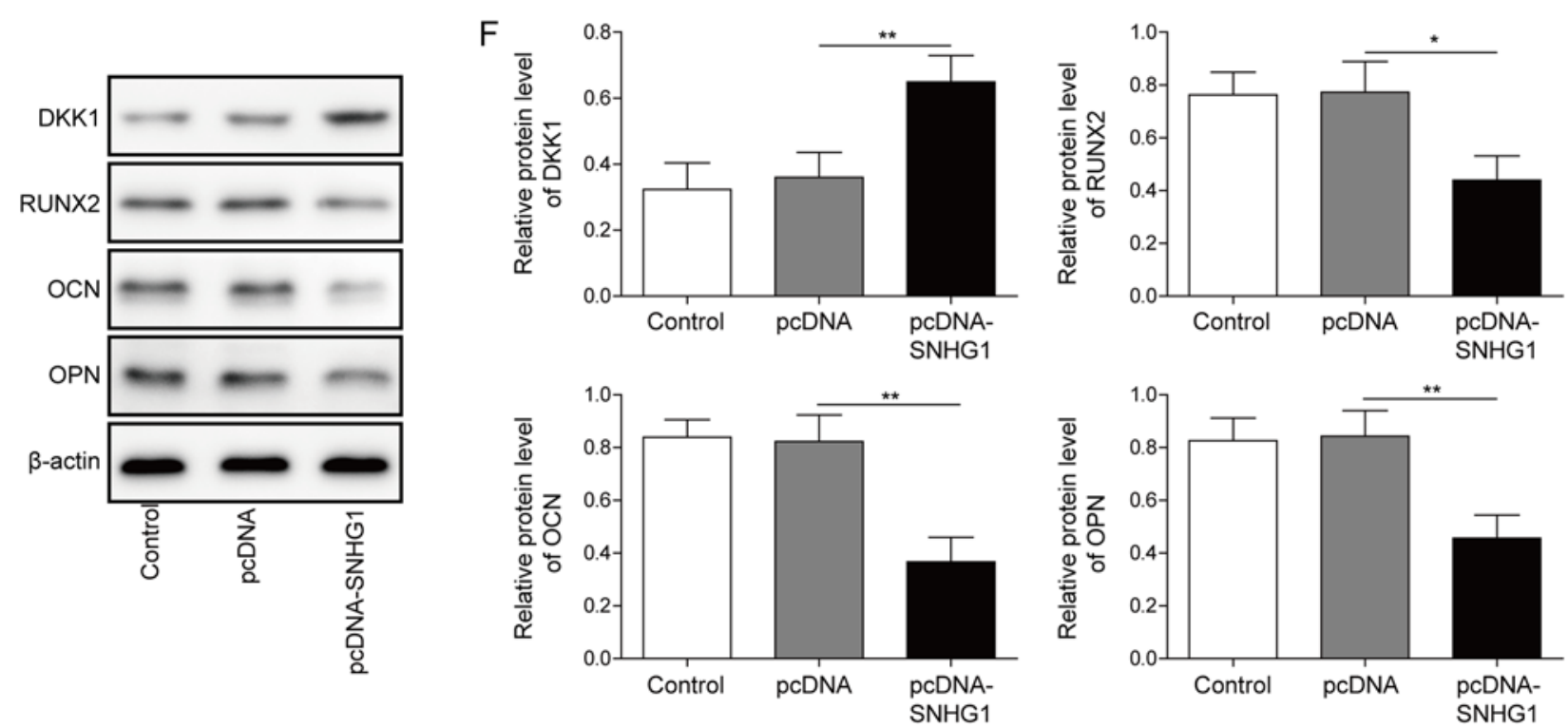

Figure 3. SNHG1 overexpression inhibits osteogenic differentiation. Effect of SNHG1 overexpression on the expression of (A) SNHG1, miR-101, DKK1, (B) RUNX2, OPN and OCN in BMSCs. Effect of SNHG1 overexpression on (C) ALP activity and (D) Alizarin Red staining in BMSCs. Protein expression levels were (E) determined by western blotting and (F) semi-quantified for DKK1, RUNX2, OPN and OCN in SNHG1-overexpression BMSCs. "P<0.05 and ${ }^{* *} \mathrm{P}<0.01$. SNHG1, small nucleolar RNA host gene 1; miR, microRNA; DKK1, dickkopf WNT signaling pathway inhibitor 1; RUNX2, RUNX family transcription factor 2; OPN, osteopontin; OCN, osteocalin; BMSC, bone mesenchymal stem cell; ALP, alkaline phosphatase.

indicated that SNHG1 may inhibit osteogenic differentiation by regulating miR-101 and DKK1.

SNHG1 inhibits BMSC osteogenic differentiation via miR-101. To verify DKK1 was the target of miR-101, BMSCs were transfected with miR-101 mimics and mimics-NC. Compared with the mimics-NC group, DKK1 expression was significantly decreased and miR-101 expression was significantly increased in the miR-101 mimics group (Fig. 4A). To assess whether SNHG1 regulated osteogenic differentiation via miR-101, BMSCs were co-transfected with pcDNA-SNHG1 and miR-101 mimics. SNHG1 overexpression significantly increased DKK1 expression compared with the pcDNA group, which was reversed by co-transfection with miR-101 mimics (Fig. 4B).
The expression levels of the osteogenesis-related markers were significantly decreased by SNHG1 overexpression compared with the pcDNA group, whereas co-transfection with miR-101 mimics reversed SNHG1 overexpression-induced effects (Fig. 4C). Furthermore, miR-101 mimics also reversed SNHG1 overexpression-mediated effects on ALP activity and matrix mineralization (Fig. 4D and E). Collectively, the results indicated that miR-101 reversed SNHG1 overexpression-mediated inhibition of osteogenic differentiation.

SNHG1 regulates the Wnt/ $\beta$-catenin signaling pathway to attenuate osteogenic differentiation. As DKK1 was identified as a target of miR-101, whether SNHG1 regulated the expression of DKK1 and proteins in its downstream signaling pathway 
A

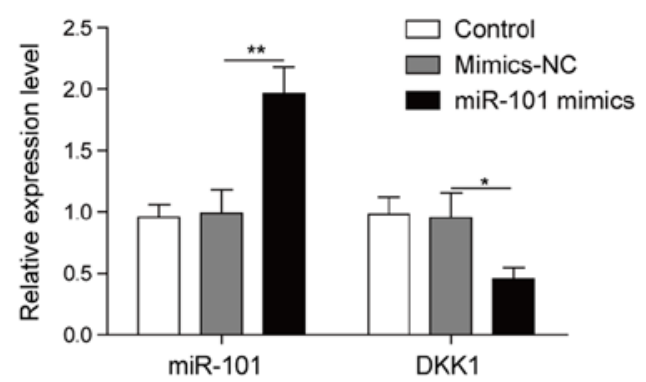

C

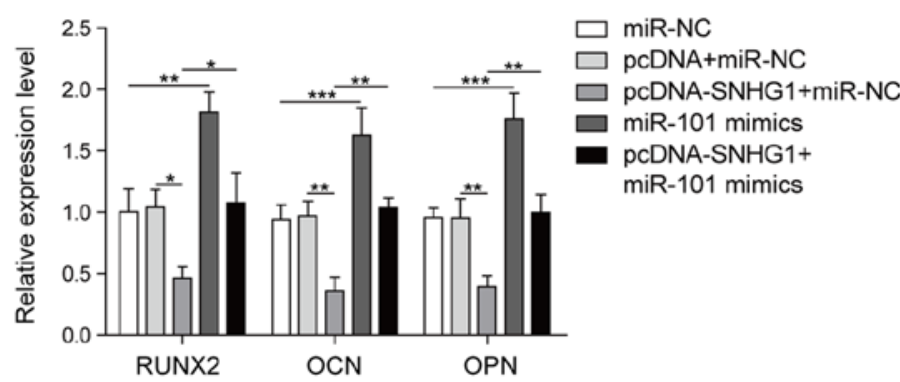

B

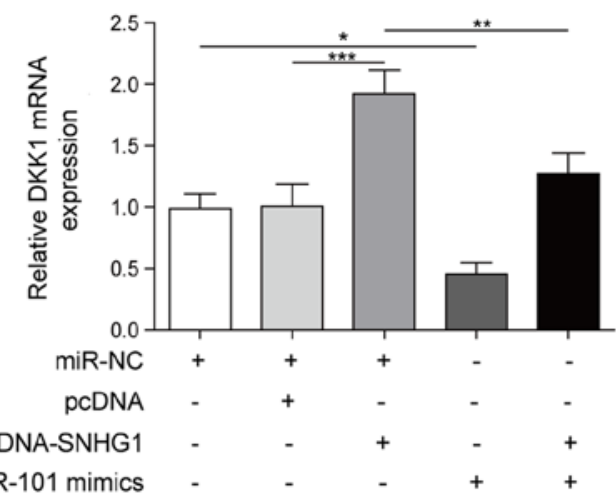

D

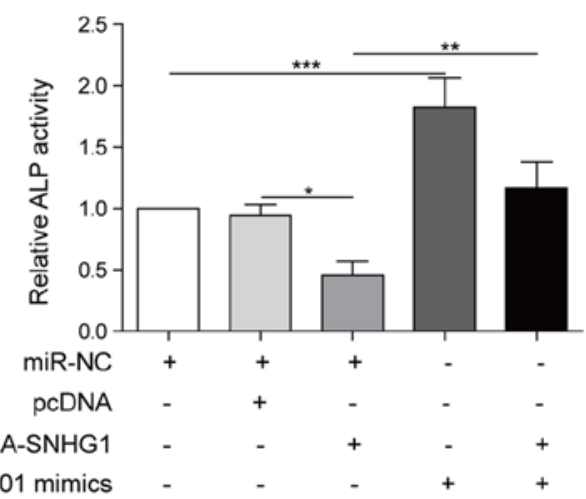

E

miR-NC pcDNA+miR-NC

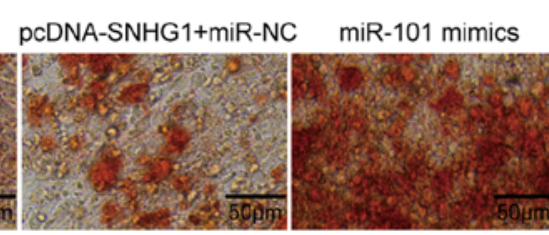

PcDNA-SNHG1+ miR-101 mimics
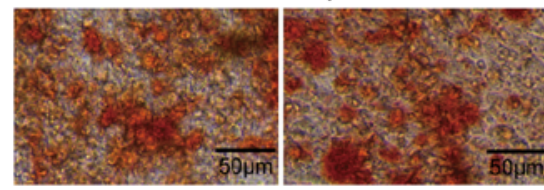

Figure 4. SNHG1 overexpression alleviates BMSC osteogenesis differentiation by targeting miR-101. (A) miR-101 and DKK1 expression levels in BMSCs transfected with miR-101 mimics and mimics-NC. (B) DKK1 expression levels in BMSCs following co-transfection with pcDNA-SNHG1 and miR-101 mimics. (C) The expression levels of RUNX2, OPN and OCN expression in BMSCs co-transfected with pcDNA-SNHG1 and miR-101 mimics. (D) ALP activity and (E) Alizarin Red staining in BMSCs co-transfected with pcDNA-SNHG1 and miR-101 mimics. ${ }^{*} \mathrm{P}<0.05,{ }^{* *} \mathrm{P}<0.01$ and ${ }^{* * * *} \mathrm{P}<0.001$. SNHG1, small nucleolar RNA host gene 1; BMSC, bone mesenchymal stem cell; miR, microRNA; DKK1, dickkopf WNT signaling pathway inhibitor 1; NC, negative control; RUNX2, RUNX family transcription factor 2; OPN, osteopontin; OCN, osteocalin.

was further examined. SNHG1 overexpression upregulated the protein expression levels of DKK1 and downregulated the protein expression levels of $\beta$-catenin, RUNX2, OCN, OPN, OSX and COLlal compared with pcDNA (Fig. 5A and B). miR-101 mimics reversed SNHG1 overexpression-mediated effects on protein expression (Fig. 5A and B), indicating that SNHG1 regulated the expression of DKK1 and its downstream Wnt/ $\beta$-catenin signaling pathway by sponging miR-101 to attenuate osteogenic differentiation.

\section{Discussion}

As a serious and complicated bone disease, OP is associated with a high risk of fracture (19). Previous studies have demonstrated that abnormal BMSC osteogenic differentiation is the leading cause of OP $(2,20,21)$. Therefore, regulation of BMSC osteogenic differentiation is a vital focus in OP pathogenesis research.

lncRNAs can serve a crucial role in the regulation of osteogenic differentiation. For example, lncRNA twist family bHLH transcription factor (TWIST)1 can promote periodontal mesenchymal stem cells from patients with periodontitis (PPDLSC) and periodontal mesenchymal stem cells from healthy microenvironment (HPDLSC) osteogenic differentiation by inhibiting the expression of TWIST (22). IncRNA-OG was reported to promote BMSC osteogenic differentiation under the regulation of heterogeneous nuclear ribonucleoprotein $\mathrm{K}$ (23). Moreover, H19 imprinted maternally expressed transcript (H19) can serve as a competing endogenous RNA (ceRNA) to promote osteogenic differentiation via the Wnt/B-catenin signaling pathway (24). Moreover, lncRNA SNHG1 can inhibit BMSC osteogenic differentiation by modulating the $\mathrm{p} 38$ MAPK signaling pathway (10). However, the downstream molecular mechanisms underlying the effects of SNHG1 in osteogenic differentiation are not completely understood. The present study indicated that SNHG1 expression was downregulated in time-dependent manner during osteogenic differentiation, and SNHG1 overexpression inhibited osteogenic differentiation, indicating a negative regulatory role of SNHG1 in BMSC osteogenic differentiation.

ceRNA was reported to be a new regulatory molecular mechanism underlying lncRNAs, where lncRNAs function as 

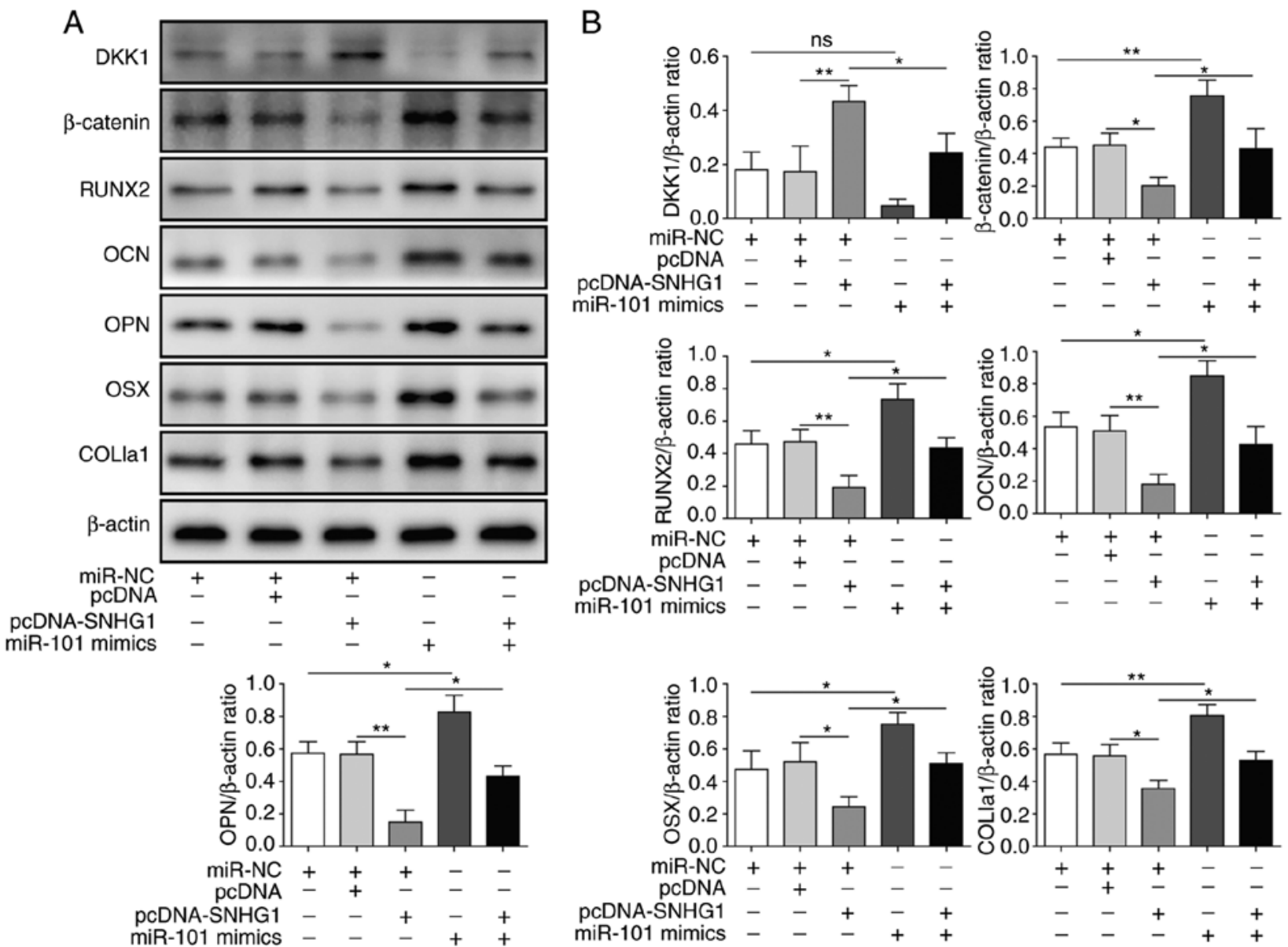

Figure 5. SNHG1 regulates the Wnt/ $\beta$-catenin signaling pathway to inhibit osteogenic differentiation. Protein expression levels were (A) determined by western blotting and (B) semi-quantified for $\beta$-catenin, DKK1, RUNX2, OCN, OPN, OSX and COL1a1 in bone mesenchymal stem cells co-transfected with pcDNA-SNHG1 and miR-101 mimics. "P $<0.05$ and $^{* *} \mathrm{P}<0.01$. SNHG1, small nucleolar RNA host gene 1; DKK1, dickkopf WNT signaling pathway inhibitor 1; RUNX2, RUNX family transcription factor 2; OCN, osteocalin; OPN, osteopontin; OSX, osterix; COL1a1, collagen type I $\alpha 1$ chain; NC, negative control.

miRNA sponges to upregulate the expression of their targets (25). SNHG1 was also reported to function as a ceRNA and serve a regulatory role with this mechanism. For instance, SNHG1 can regulate programmed cell death 4 expression by sponging miR-195-5p in hepatocellular carcinoma (26). SNHG1 can also antagonize the effect of miR-145a-5p on the downregulation of NUAK family kinase 1 in nasopharyngeal carcinoma cells (27). Besides, SNHG1 can promote osteosarcoma tumorigenesis by sponging miR-326 (28). Therefore, the present study investigated whether SNHG1 regulated osteogenic differentiation as a ceRNA. As miR-101 was predicted to be the target of SNHG1, the expression of miR-101 and its relationship with SNHG1 in BMSC osteogenic differentiation were investigated. Moreover, the results indicated that miR-101 overexpression reversed SNHG1-mediated inhibition of osteogenic differentiation, indicating that SNHG1 served as a ceRNA to regulate osteogenic differentiation by sponging miR-101. To the best of our knowledge, the present study was the first to suggest that SNHG1 regulated BMSC osteogenic differentiation by sponging miR-101 as a ceRNA.

The Wnt/ $\beta$-catenin signaling pathway was reported to be involved in osteogenic differentiation, which was also the downstream molecular mechanism underlying a number of lncRNAs (29,30). For example, IncRNA H19 could promote rat ectomesenchymal stem cell osteogenic differentiation via the Wnt/ $\beta$-catenin signaling pathway (24), whereas IncRNA KCNQ1OT1 promoted osteogenic differentiation to relieve osteolysis via Wnt/ $\beta$-catenin activation (23). In addition, lncRNA-p21 was also reported to promote mesenchymal stem cell osteogenic differentiation in the rat model of osteoporosis via the $W n t / \beta$-catenin signaling pathway (31). The present study indicated that the $\mathrm{Wnt} / \beta$-catenin signaling pathway was a downstream mechanism of the SNHG1/miR-101 axis for the regulation of osteogenic differentiation, which was consistent with previous reports.

The present study had a number of limitations. The pathogenesis of OP requires further investigation. For instance, an animal model should be applied to validate the molecular mechanism related to the $\mathrm{SNHG1/miR-101/DKK1} \mathrm{axis} \mathrm{in} \mathrm{regulation}$ of BMSC osteogenic differentiation and prove its therapeutic potential. Therefore, the mechanism identified in the present study requires further investigation using in vivo models of OP. In summary, the present study identified a potential downstream molecular mechanism of the SNHG1 axis in regulating osteogenic differentiation, providing potential therapeutic targets for OP.

\section{Acknowledgements}

Not applicable. 


\section{Funding}

The present study was supported by the Hunan Provincial Health and Family Planning Research Program (grant no. C2019121).

\section{Availability of data and materials}

The datasets used and/or analyzed during the current study are available from the corresponding author on reasonable request.

\section{Authors' contributions}

BC and JX conceptualized and designed the study and revised the manuscript. HQF collected and interpreted the data. WJF, ZX and FL analyzed and interpreted the data. FL drafted the manuscript. All authors read and approved the final manuscript.

\section{Ethics approval and consent to participate}

Not applicable.

\section{Patient consent for publication}

Not applicable.

\section{Competing interests}

The authors declare that they have no competing interests.

\section{References}

1. Iseme RA, McEvoy M, Kelly B, Agnew L, Walker FR and Attia J: Is osteoporosis an autoimmune mediated disorder? Bone Rep 7: 121-131, 2017.

2. Phetfong J, Sanvoranart T, Nartprayut K, Nimsanor N, Seenprachawong K, Prachayasittikul V and Supokawej A: Osteoporosis: The current status of mesenchymal stem cell-based therapy. Cell Mol Biol Lett 21: 12, 2016.

3. Oryan A, Kamali A, Moshiri A and Baghaban Eslaminejad M: Role of mesenchymal stem cells in bone regenerative medicine: What is the evidence? Cells Tissues Organs 204: 59-83, 2017.

4. Shen Y, Liu S, Fan J, Jin Y, Tian B, Zheng X and Fu H: Nuclear retention of the lncRNA SNHG1 by doxorubicin attenuates hnRNPC-p53 protein interactions. EMBO Rep 18: 536-548, 2017.

5. Wang CG, Liao Z, Xiao H, Liu H, Hu YH, Liao QD and Zhong D: LncRNA KCNQ1OT1 promoted BMP2 expression to regulate osteogenic differentiation by sponging miRNA-214. Exp Mol Pathol 107: 77-84, 2019.

6. Gao Y, Xiao F, Wang C, Wang C, Cui P, Zhang X and Chen X: Long noncoding RNA MALAT1 promotes osterix expression to regulate osteogenic differentiation by targeting miRNA-143 in human bone marrow-derived mesenchymal stem cells. J Cell Biochem 119: 6986-6996, 2018.

7. Sun Y, Wei G, Luo H, Wu W, Skogerbø G, Luo J and Chen R: The long noncoding RNA SNHG1 promotes tumor growth through regulating transcription of both local and distal genes. Oncogene 36 : 6774-6783, 2017.

8. Xu M, Chen X, Lin K, Zeng K, Liu X, Pan B, Xu X, Xu T, Hu X, Sun L, et al: The long noncoding RNA SNHG1 regulates colorectal cancer cell growth through interactions with EZH2 and miR-154-5p. Mol Cancer 17: 141, 2018.

9. Cui Y, Zhang F, Zhu C, Geng L, Tian T and Liu H: Upregulated lncRNA SNHG1 contributes to progression of non-small cell lung cancer through inhibition of miR-101-3p and activation of Wnt//3-catenin signaling pathway. Oncotarget 8: 17785-17794, 2017.

10. Jiang Y, Wu W, Jiao G, Chen Y and Liu H: LncRNA SNHG1 modulates p38 MAPK pathway through Nedd4 and thus inhibits osteogenic differentiation of bone marrow mesenchymal stem cells. Life Sci 228 208-214, 2019.
11. Oliveto S, Mancino M, Manfrini N and Biffo S: Role of microRNAs in translation regulation and cancer. World J Biol Chem 8: 45-56, 2017.

12. Wang H, Meng Y, Cui Q, Qin F, Yang H, Chen Y, Cheng Y, Shi J and Guo Y: MiR-101 targets the EZH2/Wnt/ $\beta$-catenin the pathway to promote the osteogenic differentiation of human bone marrow-derived mesenchymal stem cells. Sci Rep 6: 36988, 2016.

13. Deng R, Zhang J and Chen J: lncRNA SNHG1 negatively regulates miRNA-101-3p to enhance the expression of ROCK1 and promote cell proliferation, migration and invasion in osteosarcoma. Int $\mathrm{J}$ Mol Med 43: 1157-1166, 2019.

14. Yavropoulou MP and Yovos JG: The role of the Wnt signaling pathway in osteoblast commitment and differentiation. Hormones (Athens) 6: 279-294, 2007.

15. Albers J, Keller J, Baranowsky A, Beil FT, Catala-Lehnen P, Schulze J, Amling M and Schinke T: Canonical Wnt signaling inhibits osteoclastogenesis independent of osteoprotegerin. J Cell Biol 200: 537-549, 2013

16. Li Y, Lu W, King TD, Liu CC, Bijur GN and Bu G: Dkk1 stabilizes Wnt co-receptor LRP6: Implication for Wnt ligand-induced LRP6 down-regulation. PLoS One 5: e11014, 2010.

17. Mirfazeli ES, Arefian E, Nadri S, Rezazadeh Valojerdi R, Kehtari M and Zeynali B: DKK1 expression is suppressed by miR-9 during induced dopaminergic differentiation of human trabecular meshwork mesenchymal stem cells. Neurosci Lett 707: 134250, 2019.

18. Livak KJ and Schmittgen TD: Analysis of relative gene expression data using real-time quantitative PCR and the 2(-Delta Delta C(T)) method. Methods 25: 402-408, 2001.

19. Sözen T, Özışık L and Başaran NC: An overview and management of osteoporosis. Eur J Rheumatol 4: 46-56, 2017.

20. Zhou DA, Zheng HX, Wang CW, Shi D and Li JJ: Influence of glucocorticoids on the osteogenic differentiation of rat bone marrow-derived mesenchymal stem cells. BMC Musculoskelet Disord 15: 239, 2014.

21. Wang C,Meng H, Wang X,Zhao C,Peng J and Wang Y: Differentiation of bone marrow mesenchymal stem cells in osteoblasts and adipocytes and its role in treatment of osteoporosis. Med Sci Monit 22: 226-233, 2016.

22. Xu Y, Qin W, Guo D, Liu J, Zhang M and Jin Z: LncRNA-TWIST1 promoted osteogenic differentiation both in PPDLSCs and in HPDLSCs by inhibiting TWIST1 expression. Biomed Res Int 2019: 8735952, 2019.

23. Tang S, Xie Z, Wang P, Li J, Wang S, Liu W, Li M, Wu X, Su H, Cen S, et al: LncRNA-OG promotes the osteogenic differentiation of bone marrow-derived mesenchymal stem cells under the regulation of hnRNPK. Stem Cells 37: 270-283, 2019.

24. Gong YY, Peng MY, Yin DQ and Yang YF: Long non-coding RNA H19 promotes the osteogenic differentiation of rat ectomesenchymal stem cells via Wnt $/ \beta$-catenin signaling pathway. Eur Rev Med Pharmacol Sci 22: 8805-8813, 2018.

25. Du Z, Sun T, Hacisuleyman E, Fei T, Wang X, Brown M, Rinn JL, Lee MG, Chen Y, Kantoff PW and Liu XS: Integrative analyses reveal a long noncoding RNA-mediated sponge regulatory network in prostate cancer. Nat Commun 7: 10982, 2016.

26. Huang D, Wei Y, Zhu J and Wang F: Long non-coding RNA SNHG1 functions as a competitive endogenous RNA to regulate PDCD4 expression by sponging miR-195-5p in hepatocellular carcinoma. Gene 714: 143994, 2019

27. Lan $\mathrm{X}$ and Liu X: LncRNA SNHG1 functions as a ceRNA to antagonize the effect of miR-145a-5p on the down-regulation of NUAK1 in nasopharyngeal carcinoma cell. J Cell Mol Med 23: 2351-2361, 2019.

28. Wang J, Cao L, Wu J and Wang Q: Long non-coding RNA SNHG1 regulates NOB1 expression by sponging miR-326 and promotes tumorigenesis in osteosarcoma. Int J Oncol 52: 77-88, 2018.

29. Shen JJ, Zhang CH, Chen ZW, Wang ZX, Yang DC, Zhang FL and Feng KH: LncRNA HOTAIR inhibited osteogenic differentiation of BMSCs by regulating Wnt/ $\beta$-catenin pathway. Eur Rev Med Pharmacol Sci 23: 7232-7246, 2019.

30. LiN, Ma Y, Wang W, Yin CC, Wu W, Sun R,Zhao G, Li S and Wang X: LOC101928834, a novel lncRNA in Wnt//-catenin signaling pathway, promotes cell proliferation and predicts poor clinical outcome in myelodysplastic syndromes. Clin Sci (Lond) 134: 1279-1293, 2020.

31. Yang K, Tian N,Liu H, Tao XZ, Wang MX and Huang W: LncRNAp21 promotes osteogenic differentiation of mesenchymal stem cells in the rat model of osteoporosis by the Wnt//-catenin signaling pathway. Eur Rev Med Pharmacol Sci 23: 4303-4309, 2019.

This work is licensed under a Creative Commons Attribution-NonCommercial-NoDerivatives 4.0 International (CC BY-NC-ND 4.0) License. 